\title{
A Role for Conscious Accessibility in Skilled Action
}

\author{
Chiara Brozzo ${ }^{1}$ \\ Accepted: 5 November 2020 /Published online: 30 December 2020 \\ (C) The Author(s) 2020
}

\begin{abstract}
Skilled sportsmen or musicians - more generally, skilled agents — often fill us with awe with the way they perform their actions. One question we may ask ourselves is whether they intended to perform some awe-inspiring aspects of their actions. This question becomes all the more pressing as it often turns out that these agents were not conscious of some of those aspects at the time of performance. As I shall argue, there are reasons for suspecting lack of conscious access to an aspect of one's action to be incompatible with intending to perform that aspect of one's action. Subsequently, though, I will also argue that, in some cases, the incompatibility is only prima facie, and can be dispelled by drawing the following distinction: that between aspects of one's action that are merely temporarily not consciously accessed, versus aspects of one's action that are permanently inaccessible to consciousness. I will thus remove an obstacle towards saying that skilled agents intended to perform certain aspects of their actions, despite lack of conscious access to those aspects at the time of performance.
\end{abstract}

\section{Introduction}

Suppose I am a really skilled golfer and that, while standing on the green, I swing the club so that the ball is hit and lands in the hole. This is a good swing. Suppose, further, that you, while observing me, say: "Good shot! The way you oriented your right hand during the swing was really clever!" However, as it turns out, during the performance of this skilled action I was not conscious of the way I was orienting my right hand, although this was crucial for my swing turning out to be as good as it did.

Something close to this fictional example is not uncommon in the realm of skilled action. Indeed, many skilled agents are not conscious of many fundamental aspects ${ }^{1}$ of their own skilfully performed bodily actions. ${ }^{2}$

\footnotetext{
${ }^{1}$ My talk of aspects of an action is comparable to Wu's (2016) talk of features of an action.

${ }^{2}$ The focus of this article will be on skilled bodily actions, such as playing golf or playing the piano. See Christensen (2019) for other kinds of skilled action that are not necessarily tied to bodily movements.
}

Chiara Brozzo

chiara.brozzo@gmail.com

1 Philosophy Department, Durham University, 50/51 Old Elvet, Durham DH1 3HN, UK 
Here is some experimental evidence for this phenomenon. Skilled typists, the kind that type an average of 68 words per minute, were asked to type a number of four-letter words in different conditions. In some conditions, they were required to type the whole word. In other conditions, they were asked to type only the letters normally typed by - say - their right hand. Their performance was significantly slower and gave rise to significantly many more mistakes in the latter than in the former condition (Logan and Crump 2010, reported in Shepherd 2019). In Logan and Crump's words, skilled typists do not know "which hand types which letters" (Logan and Crump 2011, p. 13). Skilled typists in this experiment are a concrete example of agents who do not consciously access some aspects of their skilfully performed action: they are not conscious of which hand types which letters. When I say that skilled typists do not consciously access which hand types which letters, what I mean is the following: which hand types which letters is not part of the typists' phenomenology at the time of performance. In the rest of this article, I shall use consciously accessing and being conscious of interchangeably. ${ }^{3}$

What is the significance of lack of conscious access to some aspect of one's skilled action? One reason why this is significant is that it may seem as though lack of conscious access to an aspect of one's action is incompatible with having an intention representing that aspect of one's action (as I shall explain in Sections 2 and 3). The following challenge thus arises:

The Challenge: if an agent is not conscious of some aspect of their skilled action, can we say that this agent intended to perform that aspect of their action? ${ }^{4}$

A number of authors who have tackled this challenge have answered negatively, maintaining that lack of conscious access to some aspects of one's action may be necessitated by the fast timing with which the action is executed (see, e.g., Pacherie 2008; Papineau 2013), and that this is incompatible with the idea that the skilled agent intended to perform those aspects of their action. Those aspects of the skilled agent's action are therefore accounted for in a different way-for instance, Papineau (2013) suggests that they might be part of a raft of dispositions (in response to circumstance $C$, produce behaviour $B$ ) activated by an overall intention to, say, play defensively as opposed to aggressively. While I think that this is right in some cases, I shall argue that other cases allow for aspects of one's skilled action to be accounted for in terms of an intention representing them. In particular, in the opening example, did I intend to orient my right hand in the impressive way that you noticed during my brilliant swing? My aim is to defend the idea that, if certain conditions were in place, an obstacle is removed towards saying that I did.

\footnotetext{
${ }^{3}$ My usage of conscious access is somewhat unorthodox in the light of Block's distinction between phenomenal consciousness and access consciousness (Block 1995). In effect, when I say that someone does not consciously access a certain aspect of their action, what I mean from the point of view of Block's distinction is that they are not phenomenally conscious of that aspect.

${ }^{4}$ An anonymous reviewer raises an important point: this challenge could be undercut if one characterised the skilled agent's situation in the following way. Rather than intending to perform certain aspects of their skilled action, the agent may be merely performing certain aspects of their skilled action intentionally. If Bratman $(1984,1987)$ is right, the latter does not imply the former. So, what becomes of the challenge if a certain aspect of an action is performed intentionally, but is not intended? I contend that a version of the challenge still stands, because it can be argued that doing something intentionally, just like intending to do something, is seemingly incompatible with certain kinds of lack of conscious access to an aspect of one's action. More on this in footnote 12 .
} 
In outline, this is what I shall do in the rest of this article. First, I will argue that the possibility for conscious access to what an intention represents-say, a certain aspect of an action - is a prerequisite on having a genuine intention (as opposed to some other kind of attitude or representation) to perform that aspect of the action (Sections 2 and 3). I will then explain how this prerequisite makes lack of conscious access to a certain aspect of one's action seemingly incompatible with having an intention representing that aspect of one's action, thus giving rise to a challenge for certain skilled actions (Section 4). I will then present a distinction between kinds of lack of conscious access (Section 5). Having done this, I will apply one of the terms of this distinction to some cases of skilled action, in order to rescue them from the challenge (Section 6). I will clarify that the skilled actions that may thus be rescued by no means exhaust the set of skilled actions. Still, they are an important subset of it (Section 7).

\section{The Role of Conscious Access in Intention}

In the opening example, I described orienting my right hand in the way I did as an aspect of my skilled action. What is required for me to have an intention representing this aspect of my action? To answer this question, something about what an intention is has to be mentioned. An intention is a representation of a certain state of affairs where this state of affairs is represented with a so-called world-to-mind direction of fit (Searle 1983; see also Anscombe 1957; Mele 1992; Pacherie 2008). This means that, for an intention to be fulfilled, the world needs to conform to what the intention represents. ${ }^{5}$ To this extent, intentions are analogous to desires, which also represent states of affairs with a world-to-mind direction of fit: for a desire to be fulfilled, the world needs to conform to what the desire represents. Setting aside those who think that intentions reduce to combinations of beliefs and desires (see Sinhababu 2013 for a recent account of this kind), if there is any reason to think of intentions as distinctive attitudes, it is that intending to do something implies being committed to doing it, whereas desiring to do something does not (I can desire to win the lottery without being committed to it-I can't even be sensibly committed to it). This has been theorised by Michael Bratman (1987), in terms of the idea that intentions are subject to a normative requirement for strong consistency that I shall call the strong consistency requirement. According to it, we should not intend any course of action that conflicts with any of our other intentions or beliefs. For example, I shouldn't intend to orient my right hand in a certain way if I also believe it will be detrimental to the success of my swing, and I also intend my swing to be successful. If one's intentions do not meet this requirement, as they sometimes do (how many times have you intended to do something that you know to be in conflict with something else you intend or believe?), then, according to Bratman, one is guilty of a criticisable form of irrationality.

If my intention is to be evaluated from the point of view of rationality, a precondition on this seems to be that I should have conscious access to what my intention (and other attitudes supposed to not conflict with it) represents: I can only be held irrational for having attitudes that I know to be inconsistent. By contrast, if I am not conscious of having a certain attitude, I will not be aware of any conflict between it and other attitudes of mine.

\footnotetext{
${ }^{5}$ The direction of fit of intentions is to be contrasted with that of beliefs. Beliefs have the reverse direction of fit, namely mind-to-world: for a belief to be true, what the belief represents needs to conform to how things actually are-namely, to the world.
} 
It can therefore be argued that, in order for the strong consistency requirement to even be applicable to intentions, one should be conscious of what one intends to do. Thus, conscious access to what a certain attitude or representation represents seems to be a precondition on that attitude or representation being an intention.

\section{Too Demanding a Notion of Intention?}

The following doubt may now arise: is the notion of intention that I have just presented too demanding? For example, aren't there notions of intention that do not require conscious access to what they represent?

Conscious access to what intentions represent is a requirement that can be justified independently of Bratman's proposal. Even if one disagrees that intentions are subject to the strong consistency requirement, one can still endorse the idea that intentions have a role in getting things done. As such, they should enter relationships of motivation and constraint with other attitudes that an agent has: e.g., the desire to win a match, the belief that a certain strategy is better than another for achieving this end, and so on. One should therefore be able to form intentions in the light of, i.e. constrained and motivated by, other attitudes. For this to happen, all of these attitudes, including the intentions that the agent means to form, should be available to the agent's consciousness. This is why conscious access to an aspect of one's action is a reasonable requirement on having an intention representing that aspect.

A very noteworthy exception to this widespread requirement was provided by Pacherie (2008). At the time, Pacherie defended a threefold distinction between intentions, which included a notion of intention, namely motor intention (abbreviated in M-intention), that allowed for what motor intentions represent to be consciously inaccessible. Indeed, this notion of intention was motivated precisely, among other things, by invoking the case of skilled action: many skilled actions, Pacherie correctly pointed out, are executed at too fast a pace for the agent to consciously access the representations guiding these actions at the time of performance.

Even in the context of this proposal, Pacherie acknowledged that her notion of Mintention was somewhat unorthodox, something that has been re-emphasised more recently by Mylopoulos and Pacherie (2019). Thanks to her unorthodox proposal, however, Pacherie should be credited with insightfully bringing to the attention of philosophers representations involved in the production of actions executed at a very quick pace and often spontaneously, raising the question as to whether these representations should be considered intentions. This question had been previously answered negatively by Bach (1978). In more recent years, there has been a gradual move towards acknowledging these representations, now generally referred to as motor representations, as philosophically interesting, but, at the same time, importantly different from intentions (e.g., Butterfill and Sinigaglia 2014; cf. Shepherd 2019). ${ }^{6}$

\footnotetext{
${ }^{6}$ An interesting complication is that this is not to do exclusively, or even mainly, with the idea that what motor representations represent is not consciously accessible. Against this idea, there is evidence suggesting that what some motor representations represent is consciously accessible (Jeannerod 1994, 2006; further evidence is reported in Ferretti 2016). Butterfill and Sinigaglia (2014) argue that motor representations differ from intentions because motor representations have a different format from intentions. This has given rise to a rich debate (e.g., Levy 2017; Mylopoulos and Pacherie 2017; Ferretti and Zipoli Caiani 2019; Pavese 2019; Shepherd 2019).
} 
Therefore, if what motor representations (or at least some of them) represent is not consciously accessible, this does not constitute a counterexample to the idea that having an intention requires conscious access to what the intention represents.

At this point, though, another objection may arise. It is possible for some representations of aspects of one's action to actually be constrained by the content of other attitudes that the agent has, even though what these representations represent is not consciously accessed by the subject. For example, suppose that I adopt a certain hand configuration to grasp the golf club handle that is represented by a motor representation, $^{7}$ and that I cannot consciously access what this representation represents. Yet, what the motor representation represents, i.e. this hand configuration, is constrained by my intention-say, to swing the club in a certain way. So, in effect, consistency between the content of my intention and what the motor representation representsnamely, my hand configuration - is ensured (a point along these lines has recently been made both by Fridland 2017b and by Mylopoulos and Pacherie 2019). ${ }^{8}$

In answer to this objection, the following may be said. If we think of the strong consistency requirement as a plausible requirement on intentions, then we'll expect the subject as a whole, rather than one of its subsystems (e.g., the motor system), to be responsible for consistency among their attitudes and representations. This is so that the subject as a whole may be assessed for rationality or irrationality in holding certain attitudes. If so, then, we'll expect the subject to be able to consciously adopt their attitudes, and, as a consequence, be able to access what these attitudes represent, in order for the subject as a whole to be responsible for consistency among their attitudes.

On the other hand, if we drop the strong consistency requirement, while the notion of constraint across attitudes and representations may be made sense of regardless of whether the subject has conscious access to what these attitudes and representations represent, the notion of motivation to form certain attitudes on the basis of others is hard to make sense of in the absence of the subject having conscious access to the content of their attitudes, and consciously forming additional attitudes in the light of those they already possess. Therefore, it seems that conscious access to what a certain attitude represents is a precondition on that attitude being an intention, independently of Bratman's proposal.

\section{The Role of Conscious Access between Action and Intention}

How does being conscious of what one's intention represents bear on consciously accessing (aspects of) the action that fulfils the intention in question? This may not be obvious at first sight, since being conscious of what one's intention represents and consciously accessing (aspects of) the action that fulfils the intention in question are conceptually distinct. ${ }^{9}$ Still, relations of implication hold between them, as follows.

The main idea is that, while executing an aspect of an action represented by an intention, the subject will, at the same time, also have the intention representing that aspect of the

\footnotetext{
${ }^{7}$ Most likely, a set of motor representations. Let's talk about a hypothetical single motor representation for the sake of simplicity.

${ }^{8} \mathrm{I}$ am grateful to an anonymous reviewer for inviting me to address this point.

${ }^{9} \mathrm{I}$ am grateful to an anonymous reviewer for inviting me to clarify this connection.
} 
action, since intentions to do $A$ are generally held to be retained during $A$-ing (see, e.g., Searle 1983; Mele 1992; Pacherie 2008). Since one is executing that aspect of the action precisely because one is attempting to fulfil one's intention representing that aspect, lacking conscious access to that aspect of the action may seem to indicate that one also lacks conscious access to what the attitude representing this aspect of the action represents. ${ }^{10}$ In the previous two sections, conscious access to what an attitude or representation represents has been shown to be a precondition on that attitude or representation being an intention. Because of this, if one does not consciously access an aspect of one's action at the time of performance, it may seem as though one did not intend to do anything of the kind (although it would still be possible that this aspect of one's action was under the control of a motor representation). ${ }^{11}$ Thus, lack of conscious access to a certain aspect of one's action seems to be incompatible with having an intention representing that aspect.

Applied to cases of skilled action, you may therefore think that, if I was not conscious of orienting my right hand in a certain way (as in my opening example), this is incompatible with the idea that I intended to orient my right hand in that way. This, as anticipated, gives rise to a challenge:

The Challenge: if an agent is not conscious of some aspect of their skilled action, can we say that this agent intended to perform that aspect of their action? ${ }^{12}$

I shall now draw a distinction between kinds of lack of conscious access, in order to then argue that cases of skilled action falling into some of them may be rescued from the challenge.

\section{A Distinction: Temporary Vs. Permanent Lack of Conscious Access}

I propose to distinguish two ways in which we may lack conscious access to something. The first is what I shall call temporary lack of conscious access. I have temporary lack of conscious access to something at time $t_{l}$ if I lack conscious access to it (i.e., it is not part of my phenomenology) at $t_{l}$, but there exists a time $t_{x}$ (where $t_{x}$ is different from $\left.t_{1}\right),{ }^{13}$ at which I could consciously access it. Suppose, for example, that I form a certain

\footnotetext{
${ }^{10}$ One reason to think so is that, as part of the rationality requirements to which intentions are subject, one should also be able to know that one has fulfilled their intention. This is so as to be able to update their other intentions and beliefs accordingly and, e.g., to be capable of being held irrational if they are still attempting to fulfil an intention that has already been fulfilled. In order to know that one has fulfilled their intention, however, one should have conscious access to aspects of the corresponding action, so as to be able to know whether it has been executed.

${ }^{11}$ I emphasise seem, because the conditions of incompatibility will be refined in due course.

${ }^{12}$ Recall from footnote 4 that a potential way to undercut this challenge is to characterise skilled agents as merely intentionally doing something, rather than as intending to do something. Even so, lack of conscious access to what the skilled agent is doing seems to be incompatible with the skilled agent's intentionally doing something. Here is one reason why. Setiya (2018) points out that "when it is not the execution of a directly corresponding intention, doing A intentionally is a foreseen or desired consequence of an action that is" (my emphasis), and adds that this is a necessary condition on intentional action. That an action is either foreseen or desired by the subject implies that the subject can consciously access both its representation and the action itself - again, if we hold onto the idea that the subject is in charge of choosing their attitudes discussed in Section 3 .

${ }^{13}$ Note that, for all I have said so far, $t_{x}$ might be before or after $t_{l}$.
} 
plan. Suppose, further, that, while I haven't forgotten the plan, I am also not thinking about it at a certain time $t_{l}$, and I would be able to recall it at a different time $t_{x}$. Then, at time $t_{l}$, I have temporary lack of conscious access to my plan.

Mele and Moser have in a way anticipated the notion of temporary lack of conscious access, insofar as they observe that "[a] state of having a plan [...] can exist [...] even while absent from awareness or consciousness" (Mele and Moser 1994, p. 42). Think, for example, of the distinction between occurrent and standing mental states. A mental state that is standing, rather than occurrent, is one to which one has temporary lack of conscious access. Indeed, Mele applies the distinction between standing and occurrent mental states to intentions, noting that one can have a standing intention to fly to Corsica even while sleeping - therefore, while not currently consciously accessing this intention (Mele 2007, 2009).

The contrast case for temporary lack of conscious access is what I shall call permanent lack of conscious access. I have permanent lack of conscious access to an aspect of my action when this aspect of my action can never be part of my phenomenology.

The distinction between permanent and temporary lack of conscious access may be reminiscent of Searle's distinction between shallow and deep unconscious (Searle 1990). Searle draws this distinction in order to differentiate among unconscious neurophysiological states. Specifically, he wants to distinguish those that correspond to mental states from those that do not. In order to do so, he differentiates between "states that are in principle accessible to consciousness", which he calls shallow unconscious, and states that are "inaccessible even in principle", which he calls deep unconscious (Searle 1990, p. 588).

Searle's distinction and mine, though connected, are, however, interestingly different, and do not map onto each other neatly. States that are only temporarily inaccessible to consciousness are shallow unconscious: they are the sort of states that could in principle be consciously accessed. But it is not always the case that states that are permanently consciously inaccessible are in principle consciously inaccessible, i.e. deep unconscious. Here is why. Suppose that I have an unconscious desire to wind up a certain colleague of mine. For whichever reason, I cannot consciously access this desire of mine, and, despite all the therapy I undergo in order to bring repressed desires to light, I will never be able to consciously access it. My desire to wind up my colleague is therefore permanently consciously inaccessible. This does not mean, however, that my desire is in principle inaccessible by Searle's lights. On the contrary: qua desire, i.e. a mental state, it is the sort of state that is in principle accessible to consciousness (that is, shallow unconscious). So, temporarily inaccessible states are in principle accessible, but permanently inaccessible states are not always in principle inaccessible. They might be in principle accessible.

What is the relevance of the distinction between temporary and permanent lack of conscious access for present purposes? I will show that, when an aspect of someone's skilled action is merely temporarily not accessed, then this is potentially compatible with the agent's having an intention to perform that aspect of the action.

Here is why. An agent might temporarily lack conscious access to an aspect of their skilled action insofar as they are capable of regaining conscious access to that aspect of their action (a concrete example of how this could happen in the context of skilled action will be given in Section 6). For the moment, suffice it to note the following. Suppose I form an intention to go to Copenhagen at the end of the month. There will be 
a time - for example, just after forming the intention - in which I consciously access what this intention represents, insofar as the intention is an occurrent state of my mind. Suppose that, soon afterwards, the question as to what I shall have for dinner tonight becomes occurrent. As I am pondering over recipes and the actual contents of my fridge, my intention to go to Copenhagen switches from occurrent to standing. Assuming that I do not forget about my intention to go to Copenhagen, at any point before my trip I shall be capable of making my intention to go to Copenhagen occurrent, and therefore I will regain conscious access to what this intention represents. These switches between occurrent and standing do not threaten the status of my attitude towards going to Copenhagen as an intention: all the while, I can still be held irrational if I also believe that I should be in Stockholm in the same days in which I'm supposed to be in Copenhagen.

Aspects of an agent's skilled action might be subject to similar switches between occurrent and standing as my plan to go to Copenhagen, as will be illustrated in the next section. In cases like this, lack of conscious access to these aspects may be restored at any point. Call this kind of temporary lack of conscious access recoverable. Temporary lack of conscious access of the recoverable kind can be elucidated by drawing a distinction between the notions of conscious access on the one hand and conscious accessibility on the other hand. A subject has conscious access to a certain aspect of their action at $t_{1}$ when they are currently (i.e., at $t_{l}$ ) accessing that aspect of their action-for example, because their attention has just been drawn to this aspect. By contrast, saying that a certain aspect of an action is consciously accessible to the subject at time $t_{l}$ leaves it open whether the subject is consciously accessing that aspect at $t_{l}$. That a certain aspect of an action is consciously accessible to a subject at $t_{l}$ means that there exist a time $t_{x}$ (where it should be possible for $t_{x}$ to occur after $t_{l}$ ) at which the subject may consciously access that aspect of their action (cf. Block 1995). Saying that a subject has temporary lack of conscious access to an aspect of their action at time $t_{l}$ implies merely that the subject lacks conscious access to that aspect of the action at $t_{l}$, but leaves it open whether that aspect of the action is consciously accessible to the subject — namely, whether the subject could access it at some other time. Saying that a subject has recoverable temporary lack of conscious access to an aspect of their action at $t_{l}$ means, more specifically, that aspect of the action $i s$ consciously accessible to the subject. In particular, that aspect of the action could be accessed at a time $t_{x}$ which is after $t_{l}$ - only, it is not accessed at $t_{1}{ }^{14}$

Temporary lack of conscious access of the recoverable kind is to be contrasted with a second kind of temporary lack of conscious access, which does not work on the assumption that conscious access may be restored. On the contrary, the assumption is the following. A certain aspect of one's action is consciously accessed upon the first few performances of that action, and then, following sufficient practice, the subject loses the ability to consciously access that aspect of their action, without any possibility of recovery. Call this kind of temporary lack of conscious access non-recoverable. Non-recoverable temporary lack of conscious access to a certain aspect of one's action still qualifies as temporary on the grounds that, on previous performances of that action, the agent did consciously access that aspect.

\footnotetext{
${ }^{14} \mathrm{I}$ am grateful to an anonymous reviewer for inviting me to clarify the relationship between the notions of temporary lack of conscious access on the one hand and those of conscious access vs. conscious accessibility on the other hand.
} 
How do all these different kinds of lack of conscious access to an aspect of an action relate to the possibility of having intentions representing that aspect of the action? In particular, which kinds of lack of conscious access to an aspect of an action are compatible with having an intention representing that aspect of the action? In the next section, I will show that recoverable temporary lack of conscious access to a certain aspect of an action is compatible with this aspect being represented by an intention, thus rescuing some skilled actions from the challenge.

It is worth noting that, if one accepts Bratman's proposal, conscious access to aspects of an action that one is representing as to be brought about is not sufficient to establish that an agent has an intention representing that aspect of the action. Further conditions need to be in place - e.g., the state representing this action should be subject to the strong consistency requirement. From the point of view of Bratman's proposal, rescuing some skilled actions from the challenge will achieve the removal of an obstacle towards saying that the agent intended to perform a certain aspect of their skilled action.

\section{Conscious Accessibility: A Way out of the Challenge for some Skilled Actions}

I shall rescue some skilled actions from the challenge by arguing that agents are only temporarily not conscious of aspects of their skilled actions in a way that is recoverable, in the sense illustrated in the previous section. I will show this for cases in which an individual is not conscious of certain aspects of their skilled action at the time of performance because:

(i) the individual is not focusing their attention on those aspects at the time of performance, and/or

(ii) those aspects are automatised.

I will show that cases in which the individual is not focussing their attention on those aspects at the time of performance are ones where the individual lacks conscious access to those aspects at the time of performance, but conscious access is recoverable-in other words, those aspects are still consciously accessible to the subject. I will also show that cases in which an agent is not conscious of an aspect of their action at the time of performance because that aspect is automatised are at least compatible with temporary lack of conscious access of the recoverable kind.

\subsection{Attention}

Skilled musicians' performance is a good example of cases where an individual is not conscious of aspects of their skilled action during its execution because they are not focussing their attention on those aspects. A study by Chaffin and Logan (2006) shows exactly this. In the course of learning how to play a piano piece with a view to performing it in public, in the first stages of this process a skilled pianist will attend to so-called basic cues, concerned with low-level aspects of her bodily action, such as the way in which two consecutive keys should be pressed (e.g., legato vs. staccato). At subsequent stages of learning, the pianist will attend to cues that concern more abstract aspects of performance. Among these, Chaffin and Logan distinguish interpretive cues 
from expressive cues. Interpretive cues are supposed to bring attention to aspects such as timing and timbre, e.g. "start crescendo". Expressive cues invite the performer to attempt to convey certain feelings to the audience, e.g. "start building tension". When the pianist attends to either interpretive or expressive cues, she does not attend to basic ones, and vice versa. ${ }^{15}$ As a consequence, it may come about that some aspects of her performance, such as the posture of some of her fingers or of one of her hands, are not consciously accessed while she attends to starting building tension, and vice versa.

Suppose that this is actually the case. Now, attention being shifted across different aspects of one's action of piano playing seems to imply lack of conscious access to the aspects of the action that are not being attended to. That lack of conscious access, however, is merely temporary, because - Chaffin and Logan note - at later stages of learning, attention can be shifted back and forth between different kinds of cues. As a result, it is reasonable to infer that aspects of the pianist's action that are involved in attending to basic cues (e.g., the posture of some of the pianist's fingers or of one of her hands) are still consciously accessible, i.e. may be consciously accessed by the pianist, ${ }^{16}$ and likewise for aspects involved in attending to interpretive or expressive cues, such as "start crescendo" or "start building tension". Therefore, when lack of conscious access to an aspect of a certain action is the consequence of an attention shift, this access may be recovered. As in the case of my plan to go to Copenhagen, the pianist simply happened not to consciously access certain aspects of her action at a certain time, and these may just as well have been accessed. This is perfectly compatible with these aspects of the agent's action being represented by an intention. Thus, cases of skilled action where lack of conscious access to some of their aspects is due to attention shifts may be rescued from the challenge. Recall that the challenge ran as follows:

The Challenge: if an agent is not conscious of some aspect of their skilled action, can we say that this agent intended to perform that aspect of their action?

The challenge may initially look pressing because it generically considers the possibility that an agent is not conscious of some aspects of their skilled action, without

\footnotetext{
${ }^{15}$ An anonymous reviewer raises doubts about the truth of this phenomenon, by mentioning the possibility that a pianist could attend to expressive cues (e.g., start building tension) by means of attending to some basic cues (e.g., playing more and more staccato). If that were the case, it would not be true that attending to expressive cues prevents one from attending to basic cues. While this indeed sounds like a possibility in principle, it is not what Chaffin and Logan (2006) report. Their study looks at a pianist's reports in the course of learning a given piano piece for the purposes of performance. They note that, for example, at the end of session 17, the focus of the pianist's reports was (seemingly exclusively) on basic cues-often, specific fingerings at given points in the score. On the other hand, at the end of Session 24, namely one day in advance of the first public performance of the piece, the focus of the pianist's reports was (again, seemingly exclusively) on interpretation (e.g., crescendo at a certain point, pianissimo at another). Chaffin and Logan partially address the point raised by the reviewer by pointing out (about expressive rather than basic cues) that, at the end of the last session before the first public performance, the pianist's report also did not mention expression at all, and yet the following performance was expressive. However, they say that expression was "built into the automatic actions of the performance. But the pianist was not [...] focusing on expression as she played" (Chaffin and Logan 2006, p. 118). It is possible that the same might be the case for basic cues.

${ }^{16}$ It is possible that attending to low-level aspects of one's bodily action might make performance worse- - a phenomenon that Papineau (2013) calls the yips (more on this in Section 7). This, however, is not necessarily the case, and, even when it is, it does not make it any less true that attending to those aspects of the action is possible for the pianist.
} 
specifying whether this is because these aspects are merely temporarily not accessed at the time of performance or, rather, because the subject has permanent lack of conscious access to these aspects. However, it is only the latter case that is incompatible with a subject having an intention representing those aspects, because the rationality of the subject in performing that action cannot be assessed. The reason why aspects of an action to which an agent is not paying attention can be rescued from the challenge is because, in these cases, an agent is merely not consciously accessing those aspects at a certain time, but these aspects are still consciously accessible, so that the subject can still be assessed for rationality in choosing to execute them. That being the case, we can still say that the agent intended to perform those aspects of the action.

\subsection{Automaticity}

According to Bargh (1992), the central feature of an automatic process is autonomy, in the following sense: an automatic process is "one that, once started (and irrespective of whether it was started intentionally or unintentionally), runs to completion with no need for conscious guidance or monitoring" (Moors and De Houwer 2006, p. 301, reported in Fridland 2017a). ${ }^{17}$ Performing an action skilfully often results in some aspects of that action being automatised.

Here is an example. The Buccaneers, an American football team, for many years had the reputation of not being very good. This changed once Tony Dungy became their coach in 1996. What Dungy did, in effect, was to get his players to automatise a number of formations. Success for a team in American football usually relies on the team players knowing a high number of formations. By contrast, Tony Dungy taught his players to memorise much fewer formations than average, but to practice them over and over until what each team player had to do in the context of a single formation became automatic (Duhigg 2013). ${ }^{18}$ The automaticity of the players' behaviour makes it very likely that many aspects of their bodily actions - e.g., their orienting their limbs in a specific way — would not be consciously accessed as the game unfolds. ${ }^{19}$ Does this mean that those aspects of the players' actions are permanently consciously inaccessible?

It does not. The reasons for this fall into two different cases: either conscious access to these aspects may be recovered, or it may not. In order to discuss these two possibilities, let me go back to the example of the skilled typists discussed by Logan and Crump (2011).

Logan and Crump's results show that skilled typists do not know which hand types which letters. Logan and Crump interpret these results in terms of the idea that, in the

\footnotetext{
${ }^{17}$ Defining automaticity is much more complicated than this, as noted by Fridland (2017a). While acknowledging the complexity of the topic, I adopt the reported definition because it is sufficient for the purposes of the present discussion.

18 This may be well explained by a model proposed by Papineau (2013), according to which all the players had to do was to select the intention to play according to a certain formation, and what they did from then onwards unfolded automatically. According to Papineau (2013), this is due to the way their basic action control system works once it has been re-set by a given intention (see Fridland (2017b) for reservations about Papineau's model).

${ }^{19}$ Wayne Wu's distinction between aspects of one's action that are automatic as opposed to others being controlled is also relevant here: the idea is that the feature of the players' actions consisting in playing according to a certain formation is controlled, whereas the feature of orienting one's leg in a certain way is automatic (Wu 2016).
} 
control of the typists' actions, two independent loops (as they call them) develop. One is responsible for word choice, and another for how the typing of the word is subdivided between the two hands. According to Logan and Crump, the first loop is under conscious control, but the second is not. This interpretation may be further elaborated in two different ways, and I will show that at least one of them is compatible with merely temporary lack of conscious access of the recoverable kind.

The first way to elaborate Logan and Crump's interpretation is that which hand types which letters is recoverable for conscious access. ${ }^{20}$ Evidence for this would be that, upon request, typists could go back to consciously selecting which hand types which letters, even though this would be effortful. If so, it would appear that this aspect of their action is merely temporarily not accessed in a recoverable way-that is, it's still consciously accessible. In this case, as with that of attention shifts tackled in the previous subsection, their lack of conscious access to that aspect of their action would be merely contingent, and thus perfectly compatible with this aspect being represented by an intention.

The second way to elaborate Logan and Crump's interpretation, by contrast, is that the aspects of the typists' actions controlled by the second loop are no longer consciously accessible: skilled typists can no longer consciously select which hand types which letters. This option cannot be treated in the same way as the previous one. As in the case of the Buccaneers trained by Dungy, we may assume that skilled typists started their training by consciously selecting, and therefore consciously accessing, which hand types which letters. However, by the end of their training, according to this second way of elaborating Logan and Crump's interpretation, typists lost access to this aspect of their action. Admittedly, when lack of conscious access is temporary but nonrecoverable, it is at best not clear that it could be compatible with representation by an intention. Therefore, right now I can only conclude that aspects of skilled action that are not consciously accessed because they have been automatised may be rescued from the challenge when they are temporarily not accessed in a recoverable way.

\section{How Much Skilled Action Is Consciously Accessible?}

Here is an issue for my proposal. Many current philosophical accounts of skilled action work on the assumption that certain aspects of one's skilled action are permanently consciously inaccessible, for precisely some of the reasons that Pacherie (2008) originally pointed out: many skilled actions are executed at a very quick pace- - for example, in order to be able to respond appropriately to a certain stimulus in an effective way given time constraints. Many skilled actions are therefore not only quickly executed, but also spontaneous. Bratman (1987) even doubts that aspects of these actions should be classified as intentional.

Working on the assumption that some aspects of an agent's skilled action are permanently consciously inaccessible, many current philosophical proposals aim to account for these aspects of the action not as represented by intentions, but still as part of the agent's strategy in some way — e.g., because they are part of a raft of dispositions

\footnotetext{
${ }^{20}$ This is in line with what Fridland (2017a, p. 4343) reports: "Sheets-Johnstone (2012) and Tzelgov et al. (1997) have both pointed out the implausibility of agents remaining necessarily unaware of their skilled, habitual, automatic behaviors."
} 
that are activated by an overarching intention (Papineau 2013), or because they are in some sense intelligent-for example, they are sensitive to an overarching intention (Fridland 2017b). In the light of this, is the idea that an important class of aspects of skilled action is temporarily consciously inaccessible in a recoverable way just off the mark?

In the light of this question, my proposal requires qualification. I acknowledge that, indeed, many aspects of skilled action won't be represented by intentions precisely because they are permanently consciously inaccessible. My aim in this article has not been to deny this, but rather to point out that this should not obscure the fact that some aspects of skilled action are only temporarily consciously inaccessible, and in a recoverable way. This is especially likely with those aspects of one's skilled action that the agent can afford to reflect on as they unfold without damaging performance. Piano playing is a good example: as pointed out by Chaffin and Logan (2006), this is the sort of skilled action where agents can afford to change the focus of their attention multiple times, therefore consciously accessing different aspects of their action at different times, without any detriment to performance.

More generally, that there are indeed aspects of skilled action that are only temporarily consciously inaccessible is evidenced by the phenomenon that David Papineau refers to as the yips, namely starting to overthink aspects of one's skilled action in advance of performance. About the yips, Papineau notes:

It is striking that the yips arise only in connection with those sporting movements that are triggered by the players themselves, as opposed to those that are responses to their competitors' actions. It is specifically when you need to initiate some movement yourself that you are in danger of thinking about the movements you must perform. When somebody else is in control of the timing and direction of an approaching ball or other trigger to your movement, you have no time to think about what you must do - you just do it. (2013, p. 181)

In short, while my proposal is not suited to the entire realm of skilled action, it might be useful for bringing back into focus aspects of skilled action where conscious access, though missing some of the time, may nevertheless be restored.

\section{Conclusion}

Recall the opening example, in which I execute a fantastic golf swing partly thanks to the clever way in which I oriented my right hand. I pointed out that the idea that this aspect of my action is represented by an intention is challenged by the fact that, at the time of action performance, I was not conscious of orienting my hand in this clever way-i.e., I did not consciously access this aspect of my action. I have shown two cases in which this aspect of my action may be rescued from the challenge. Both cases work on the assumption that this aspect of my action was merely temporarily not accessed, rather than permanently inaccessible, and in a way that is recoverable. In other words, this aspect of my action, while not consciously accessed at the time of performance, was still consciously accessible. 
Thus, I have shown how some aspects of our skilled actions may be represented by an intention, even when we do not consciously access those aspects at the time of performance. This by no means rescues all cases of skilled action from the challenge, but the cases that may be rescued through my proposal still range far and wide in the realm of skilled action.

Acknowledgements I would like to thank Denis Buehler, Wayne Christensen, Elvira Di Bona, Naomi Eilan, Ellen Fridland, Thor Grünbaum, Al Mele, Myrto Mylopoulos, Krisztina Orbán, Elisabeth Pacherie, David Papineau, Sarah Robins, Johannes Roessler, Joshua Shepherd, Susanna Siegel, Hong Yu Wong, four anonymous reviewers, and the audiences of the conference of the European Society for Philosophy and Psychology in Athens (2019) and of the Philosophy of Neuroscience Research Forum at the University of Tübingen (2019) for inspiration and feedback. My research was supported by the John Templeton Foundation (ACT Fellowship awarded to Hong Yu Wong).

Open Access This article is licensed under a Creative Commons Attribution 4.0 International License, which permits use, sharing, adaptation, distribution and reproduction in any medium or format, as long as you give appropriate credit to the original author(s) and the source, provide a link to the Creative Commons licence, and indicate if changes were made. The images or other third party material in this article are included in the article's Creative Commons licence, unless indicated otherwise in a credit line to the material. If material is not included in the article's Creative Commons licence and your intended use is not permitted by statutory regulation or exceeds the permitted use, you will need to obtain permission directly from the copyright holder. To view a copy of this licence, visit http://creativecommons.org/licenses/by/4.0/.

\section{References}

Anscombe. 1957. G.E.M., Intention (Second Edition). Oxford: Basil Blackwell.

Bach, K. 1978. A representational theory of action. Philosophical Studies: An International Journal for Philosophy in the Analytic Tradition 34 (4): 361-379.

Bargh, J.A. 1992. The ecology of automaticity: toward establishing the conditions needed to produce automatic processing effects. The American Journal of Psychology 105 (2): 181-199. https://doi. org/10.2307/1423027.

Block, N. 1995. On a confusion about a function of consciousness. Behavioral and Brain Sciences 18 (2): $227-247$.

Bratman, M. 1987. Intention, plans, and practical reason. Cambridge: Harvard University Press.

Butterfill, S.A., and C. Sinigaglia. 2014. Intention and motor representation in purposive action. Philosophy and Phenomenological Research 88 (1): 119-145.

Chaffin, R., and T. Logan. 2006. Practicing perfection: How concert soloists prepare for performance. Advances in Cognitive Psychology 2 (2-3): 113-130.

Christensen, W. 2019. Skilled action. Philosophy Compass 14 (11): e12631.

Duhigg, C. 2013. The power of habit: Why we do what we do and how to change. London: Random House.

Ferretti, G. 2016. Through the forest of motor representations. Consciousness and Cognition 43: 177-196.

Ferretti, G., and S. Zipoli Caiani. 2019. Solving the interface problem without translation: The same format thesis. Pacific Philosophical Quarterly 100 (1): 301-333.

Fridland, E. 2017a. Automatically minded. Synthese 194 (11): 4337-4363.

Fridland, E. 2017b. Skill and motor control: Intelligence all the way down. Philosophical Studies 174 (6): 1539-1560.

Jeannerod, M. 1994. The representing brain: Neural correlates of motor intention and imagery. Behavioral and Brain Sciences 17 (2): 187-202.

Jeannerod, M. 2006. Motor cognition: What actions tell the self. New York: Oxford University Press.

Levy, N. 2017. Embodied savoir-faire: Knowledge-how requires motor representations. Synthese 194 (2): 511-530.

Logan, G.D., and M.J. Crump. 2010. Cognitive illusions of authorship reveal hierarchical error detection in skilled typists. Science 330 (6004): 683-686. 
Logan, G.D., and M.J. Crump. 2011. Hierarchical control of cognitive processes: The case for skilled typewriting. Psychology of Learning and Motivation-Advances in Research and Theory 54: 1-19.

Mele, A.R. 1992. Springs of action: Understanding intentional behavior. New York: Oxford University Press.

Mele, A.R. 2007. Persisting intentions. Noûs 41 (4): 735-757.

Mele, A.R. 2009. Effective intentions: The power of conscious will. New York: Oxford University Press.

Mele, A.R., and P.K. Moser. 1994. Intentional action. Nous 28 (1): 39-68.

Moors, A., and J. De Houwer. 2006. Automaticity: A theoretical and conceptual analysis. Psychological Bulletin 132 (2): 297-326.

Mylopoulos, M., and E. Pacherie. 2017. Intentions and motor representations: The interface challenge. Review of Philosophy and Psychology 8 (2): 317-336.

Mylopoulos, M., and E. Pacherie. 2019. Intentions: The dynamic hierarchical model revisited. Wiley Interdisciplinary Reviews: Cognitive Science 10 (2): e1481.

Pacherie, E. 2008. The phenomenology of action: A conceptual framework. Cognition 107 (1): 179-217.

Papineau, D. 2013. In the zone. Royal Institute of Philosophy Supplements 73: 175-196.

Pavese, C. 2019. The psychological reality of practical representation. Philosophical Psychology 32 (5): 784 821.

Searle, J.R. 1983. Intentionality: An essay in the philosophy of mind. Cambridge: Cambridge University Press.

Searle, J.R. 1990. Consciousness, explanatory inversion, and cognitive science. Behavioral and Brain Sciences 13 (4): 585-596.ì.

Setiya, K. 2018. Intention. Stanford Encyclopedia of Philosophy. https://plato.stanford.edu/entries/intention/. Accessed 7 Dec 2020.

Sheets-Johnstone, M. 2012. Kinesthetic memory: Further critical reflections and constructive analyses. In Body, metaphor, memory and movement, ed. S.C. Koch, T. Fuchs, M. Summa, and C. Müller, 43-72. Amsterdam: John Benhamins Publishing.

Shepherd, J. 2019. Skilled action and the double life of intention. Philosophy and Phenomenological Research 98 (2): 286-305.

Sinhababu, N. 2013. The desire-belief account of intention explains everything. Nô̂s 47 (4): 680-696.

Tzelgov, J., Z. Porat, and A. Henik. 1997. Automaticity and consciousness: Is perceiving the word necessary for reading it? The American Journal of Psychology 110 (3): 429-448.

Wu, W. 2016. Experts and deviants: The story of agentive control. Philosophy and Phenomenological Research 93 (1): 101-126.

Publisher's Note Springer Nature remains neutral with regard to jurisdictional claims in published maps and institutional affiliations. 